\title{
Using machine learning to predict the number of alternative solutions to a minimum cardinality set covering problem
}

\author{
Brooks Emerick, Yun Lu, Francis J. Vasko* \\ Department of Mathematics, Kutztown University, Kutztown, USA \\ bemerick@kutztown.edu; lu@kutztown.edu; vasko@kutztown.edu \\ *Corresponding Authors: vasko@kutztown.edu
}

\section{ARTICLE INFO}

\section{Keywords}

Minimum cardinality set

covering problem;

Unicost set covering

problem;

Machine learning;

Regression trees;

Number of alternative

optimal solution.

\section{Article history}

Received:

October 15, 2020

Revised:

January 3, 2021

Accepted:

January 15, 2021

Available online:

February 24, 2021

\section{ABSTRACT}

Although the characterization of alternative optimal solutions for linear programming problems is well known, such characterizations for combinatorial optimization problems are essentially non-existent. This is the first article to qualitatively predict the number of alternative optima for a classic NP-hard combinatorial optimization problem, namely, the minimum cardinality (also called unicost) set covering problem (MCSCP). For the MCSCP, a set must be covered by a minimum number of subsets selected from a specified collection of subsets of the given set. The MCSCP has numerous industrial applications that require that a secondary objective is optimized once the size of a minimum cover has been determined. To optimize the secondary objective, the number of MCSCP solutions is optimized. In this article, for the first time, a machine learning methodology is presented to generate categorical regression trees to predict, qualitatively (extra-small, small, medium, large, or extra-large), the number of solutions to an MCSCP. Within the machine learning toolbox of MATLAB®, 600,000 unique random MCSCPs were generated and used to construct regression trees. The prediction quality of these regression trees was tested on 5000 different MCSCPs. For the 5output model, the average accuracy of being at most one off from the predicted category was $94.2 \%$.

\section{Introduction}

Characterizing and determining alternative optimal solutions to linear programming problems is a standard topic in operations research textbooks (Hillier \& Lieberman, 2010; Taha, 2017). However, the literature on alternative optimal solutions for combinatorial optimization problems, especially NP-hard combinatorial optimization problems, is virtually non-existent. The only paper the authors are aware of is by Huang et al. (2018), which tries to find multiple solutions for the traveling salesman problem by incorporating a genetic algorithm into a niching technique. Papers that come close to characterizing alternative optimal solutions do not deal with NP-hard problems. For example, Hamacher \& Queyranne (1985) developed an algorithm based on a binary search tree procedure to find the $\mathrm{K}$ best bases in a matroid, perfect matchings, and best cuts in a network. 
Lawler (19720 presented a system for computing the $K$ best solutions to discrete optimization problems and then applied it to the shortest path problem. To the authors' knowledge, there are no procedures presented in the literature for either quantitatively or qualitatively predicting how many alternative optimal solutions there are for any combinatorial optimization problem. This is the first article to develop a methodology for predicting qualitatively the number of alternative optimal solutions to an NP-hard combinatorial optimization problem, namely, the minimum cardinality set covering problem (MCSCP). The mathematical formulation of the MCSCP will now be given.

Let $A=\left[a_{i j}\right]$ be an $m \times n$ matrix, where $m<n$, and the entries of $A$ are zeros and ones. Suppose the row and column sum of the matrix $A$ is at least one. We seek the solution to the minimum cardinality set covering problem (MCSCP), which is formulated as follows: Let $x=\left[x_{j}\right]$ be an $n \times 1$ column vector of ones and zeros only ( $x$ is a bit string), then

$$
\begin{cases}\text { Minimize: } & \sum_{j=1}^{n} x_{j} \\ \text { Constraint: } & \sum_{j=1}^{n} a_{i j} x_{j} \quad \text { for } i=1,2, \cdots, m\end{cases}
$$

For any matrix $A$ that meets the above conditions, there is at least one solution to the MCSCP, which implies that the solution vector $x$ may not be unique. In this article, the focus is on answering the following question: Given the matrix $A$ with known density of ones, can the number of alternative solution vectors $x$ with the same minimum cardinality be confidently predicted?

Although the minimum cardinality set covering problem (MCSCP) is NP-hard (Karp, 1972), with recent improvements in integer programming software (Bixby, 2012) it is now possible to determine optimal solutions to some "larger" MCSCPs in a reasonable amount of time. Hence, some industrial applications involving MCSCPs can be solved exactly. Furthermore, there are essential industrial applications that are essentially pre-emptive goal programs in which the first goal is to solve an MCSCP and then, given the MCSCP solution, find an optimal solution to a secondary objective. Two such examples from the steel industry are optimal ingot mold selection (Vasko et al., 1987) and metallurgical grade assignment (Vasko et al., 1989). For ingot mold selection, the first priority is to minimize the number of mold sizes because the inventory investment, material-handling, and logistical considerations associated with an additional mold size outweigh the potential yield or productivity benefits from increasing the number of mold sizes. Similar arguments can be made to keep the number of metallurgical grades assigned to customer orders to a minimum. For these applications, knowing, at least qualitatively, the number of alternative optimal MCSCP solutions could help determine the appropriate approach to use when solving the optimum value of the secondary objective (yield loss for ingot mold selection and material costs grade assignment).

The authors were familiar with the work of Vasko et al. (2005) in which regression tree analysis was used to qualitatively predict if coal blends would be good or bad for coke oven processes and blast furnace operations. There were four possible outcomes: bad coke oven-bad blast furnace impact, bad coke oven-good blast furnace impact, good coke oven-bad blast furnace impact, and 
good coke oven-good blast furnace impact. For the candidate coal blend to receive further consideration, the regression tree model predicted that its use would result in a good coke oven and good blast furnace impact. Furthermore, Saleh et al. (2018) successfully used artificial neural networks (ANN), which is considered a subset of machine learning, to predict $\mathrm{CO}_{2}$ emissions. Additionally, Williams et al. (2009) used ANNs in MATLAB to successfully solve a mass spectrometry application. Given these successful applications, the authors decided to use the Statistical and Machine Learning Toolbox function fitter in MATLAB to generate regression trees to predict the number of alternative optimal solutions to MCSCPs qualitatively.

This paper is organized as follows: In Section 2 we present our methodology, which consists of statistical analysis on a representative set of MCSCPs, the construction of regression trees trained from this set, and the validation of the regression trees on a test set of MCSCPs; in Section 3 , we discuss the implications and limitations of our results; and we close with concluding remarks in Section 4.

\section{Research Methodology}

To qualitatively predict the number of alternative solutions to a given MCSCP, we use a methodology based on machine learning. We study the characteristics of a large sample of MCSCPs to identify relevant attributes of a particular problem that may suggest a number of alternative solutions to that problem. Because a single MCSCP is completely determined by the constraint matrix $A$, we randomly generate 600,000 matrices in MATLAB to act as a representative sample. Each matrix was unique with a fixed size and density of ones. Specifically, each matrix

$A=\left[a_{i j}\right]$ has $m=10$ rows, $n=20$ columns, and $p=20 \%$ density of ones. It is an initial study, and we hope to adapt our results to various sizes and thicknesses in the future. Below, we describe our methods for statistically analyzing this representative sample of MCSCPs. Then, we describe how the sample is used as a training set to generate several categorical regression trees, which are then used to predict the number of alternative optima of any given MCSCP.

\subsection{Statistical Analysis}

We seek a frequency distribution of the number of alternative optimal solutions for each of the 600,000 MCSCPs. To this end, we solve each MCSCP using the intlinprog function of MATLAB. Then, we use a brute force method to enumerate all alternative solutions. For example, if the minimum cardinality of an MCSCP is 4 , then we search through all ${ }_{20} C_{4}$ sets of columns of size 4 to determine if that set is indeed a solution. Given the number of alternative solutions for each MCSCP, the purpose of the analysis is to identify specific characteristics of the matrix $A$ that correspond to a higher or lower number of alternative solutions.

The descriptive statistics for the number of optimal solutions for the 600,000 MCSCPs is given in Table 1. The table provides the mean, standard deviation, and the five-number summary of the entire data set. The data set has a strong right skew. Table 1 indicates that the minimum number of optimal solutions is one, which means a matrix yields a unique solution. The maximum number of alternative solutions is 672 . The table shows the outlier threshold, which is the maximum number of optimal solutions not considered an outlier. In this case, if a matrix has greater than or equal to 36 optimal solutions, then it is considered an outlier relative to the data set. 
Table 1. Summary statistics for the number of optimal solutions of the global data set of MCSCPs

\begin{tabular}{|c|c|c|c|c|c|c|c|c|c|}
\hline \multirow{2}{*}{ Mean } & \multirow{2}{*}{ Std. Dev. } & \multicolumn{5}{|c|}{ Five-Number Summary } & $\begin{array}{c}\text { Outlier } \\
\text { Threshold }\end{array}$ & $\begin{array}{c}\text { Proportion } \\
\text { in } \boldsymbol{U}_{\text {min }}\end{array}$ & $\begin{array}{c}\text { Proportion } \\
\text { in } \boldsymbol{U}_{\text {max }}\end{array}$ \\
\cline { 3 - 8 } & & Min & $\boldsymbol{Q}_{\boldsymbol{L}}$ & $\mathbf{M}$ & $\boldsymbol{Q}_{\boldsymbol{L}}$ & Max & & & \\
\hline 13.79 & 20.62 & 1 & 2 & 6 & 15.5 & 672 & 35.75 & $14.93 \%$ & $10.31 \%$ \\
\hline
\end{tabular}

An MCSCP (matrix) is defined to have an unusually large number of solutions if the number of solutions is an outlier, i.e., exceeds the value of 35.75 . There are $61,846(10.31 \%)$ MCSCPs with an unusually large number of solutions, and so these MCSCPs are denoted as $U_{\max }$. The set $U_{\min }$ is defined as the set of all MCSCPs with exactly one optimal solution. There are precisely 89,604 MCSCPs (14.93\%) with a unique solution vector of the entire data set.

For each of the 600,000 MCSCPs, the goal was to identify important (relevant to the number of alternative solutions) characteristics of the corresponding $A$ matrix. Observe that the minimum cardinality and the number of alternative solutions is invariant under row or column swapping. Figure 1 shows a matrix $A$ with minimum cardinality of 5 in its original form and in its sorted form, where a blue dot represents a one, and the rest of the elements are zero. To obtain this sorted form, the rows are first sorted by the first non-zero element that appears. A secondary sort is applied such that the columns are sorted by descending column sum with a tiebreaker being determined by the smallest row with a nonzero entry. Furthermore, the matrix in Figure 1 has a unique solution, which is shown as the five highlighted columns.

In the sorted form, several defining attributes of the matrix $A$ are characteristic of other matrices within the set $U_{\min }$. The matrix $\mathrm{A}$, which has a unique solution, in its original form (top) and in its sorted form (bottom). A blue dot represents a one, the rest of the matrix entries are zero. The five red columns show the solution to the MCSCP, which contains the five columns contained in the set covering. For example, in Figure 1, it should be noted that rows 4, 8, and 10 contain a single element. Because of this, all solution vectors must contain columns 4, 15, and 20 . Therefore, this matrix will have a smaller number of optimal solutions simply because three of the five possible columns in a solution must be columns 4,15 , and 20 . In short, the number of possible optimal solutions has been reduced from ${ }_{20} C_{5}=15504$ to ${ }_{17} C_{2}=136$. This is a $99 \%$ reduction. Thus, the number of rows of the matrix $A$ that contain a single element is a matrix characteristic of interest to this analysis. Furthermore, it should be noted that column 4 also has only a single element. In contrast, columns 15 and 20 contain other elements that may contribute in some way to a covering. Because column 4 must be in the solution, but it doesn't "help" with a covering, this matrix is said to have an isolated point. Hence, the number of isolated points in any matrix is also a characteristic of interest to this analysis.

Using the sorted version of each matrix as a visual guide, we compute descriptive statistics for other attributes about matrices' samples. Table 2 compares a few other defining characteristics of the matrices with a unique solution (the set $U_{\min }$ ) to the matrices with an unusually large number of solutions (the set $U_{\max }$ ). The average and standard deviation of the measures for each set are compared. In addition to the number of single element rows and isolated points, the number of duplicate columns, the number of dominated and dominating columns, the proportion of nonzero elements in the matrix $A A^{T}$, the number of elements in each quadrant of the sorted matrix and 
some statistical measures about the row sum and column sum is computed. In total, 26 characteristics are considered as the set of decision variables for the regression tree.
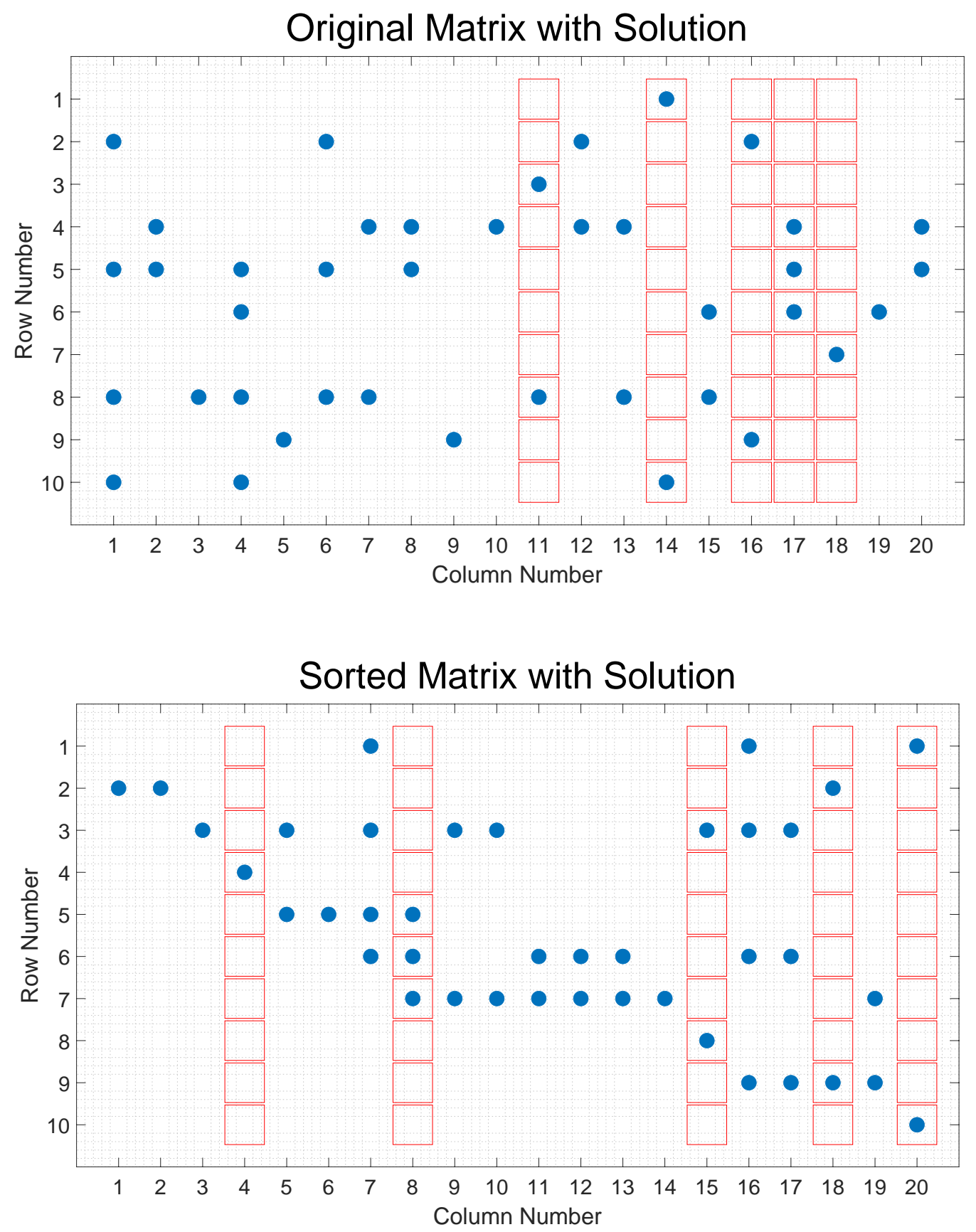

Figure 1. The matrix $A$ 
Table 2. A comparison of matrix characteristics from the set of all matrices

\begin{tabular}{|c|c|c|c|}
\hline Decision Variable & Notation & Mean/STD of $U_{\min }$ & Mean/STD of $U_{\max }$ \\
\hline Minimum Cardinality & $m$ & $3.58 / 0.51$ & $5.01 / 0.25$ \\
\hline Dominated Columns & $x_{1}$ & $10.64 / 1.76$ & $10.21 / 1.62$ \\
\hline Dominating Columns & $x_{2}$ & $12.97 / 1.07$ & $12.79 / 1.16$ \\
\hline Single Element Rows & $x_{3}$ & $0.64 / 0.69$ & $0.42 / 0.57$ \\
\hline $\begin{array}{l}\text { Maximum Column Sum of Single } \\
\text { Element Row }\end{array}$ & $x_{4}$ & $1.44 / 1.61$ & $1.01 / 1.42$ \\
\hline Isolated Points & $x_{5}$ & $0.10 / 0.31$ & $0.06 / 0.24$ \\
\hline Duplicate Columns & $x_{6}$ & $2.56 / 1.22$ & $2.52 / 1.22$ \\
\hline Rows with Maximum Row Sum & $x_{7}$ & $1.45 / 0.81$ & $1.44 / 0.78$ \\
\hline $\begin{array}{l}\text { Proportion of Nonzero Elements in } \\
A A^{T}\end{array}$ & $x_{8}$ & $0.52 / 0.06$ & $0.48 / 0.05$ \\
\hline Maximum Eigenvalue of $A A^{T}$ & $x_{9}$ & $12.53 / 1.15$ & $12.45 / 1.15$ \\
\hline Elements in Northwest Quadrant & $x_{10}$ & $12.03 / 2.35$ & $12.25 / 2.39$ \\
\hline Elements in Northeast Quadrant & $x_{11}$ & $7.97 / 2.18$ & $7.74 / 2.14$ \\
\hline Elements in Southwest Quadrant & $x_{12}$ & $4.99 / 2.35$ & $5.13 / 2.24$ \\
\hline Elements in Southeast Quadrant & $x_{13}$ & $15.01 / 1.78$ & $14.88 / 1.89$ \\
\hline Mean of Row Sum & $x_{14}$ & $4.00 / 0.00$ & $4.00 / 0.00$ \\
\hline Standard Deviation of Row Sum & $x_{15}$ & $1.74 / 0.39$ & $1.70 / 0.34$ \\
\hline Median of Row Sum & $x_{16}$ & $3.91 / 0.38$ & $3.87 / 0.37$ \\
\hline Interquartile Range of Row Sum & $x_{17}$ & $1.82 / 0.86$ & $1.76 / 0.80$ \\
\hline Minimum of Row Sum & $x_{18}$ & $1.52 / 0.59$ & $1.65 / 0.55$ \\
\hline Maximum of Row Sum & $x_{19}$ & $6.91 / 1.03$ & $6.89 / 0.98$ \\
\hline Mean of Column Sum & $x_{20}$ & $2.00 / 0.00$ & $2.00 / 0.00$ \\
\hline Standard Deviation of Column Sum & $x_{21}$ & $1.02 / 0.16$ & $0.95 / 0.14$ \\
\hline Median of Column Sum & $x_{22}$ & $1.95 / 0.18$ & $1.98 / 0.10$ \\
\hline Interquartile Range of Column Sum & $x_{23}$ & $1.60 / 0.46$ & $1.54 / 0.54$ \\
\hline Minimum of Column Sum & $x_{24}$ & $1.00 / 0.00$ & $1.00 / 0.00$ \\
\hline Maximum of Column Sum & $x_{25}$ & $4.29 / 0.75$ & $4.02 / 0.65$ \\
\hline
\end{tabular}

The bolded entries in the table represent the variables that yield the top five largest $t$-statistics for a difference of means significance test. To clarify, most of the means (for each decision variable) are significantly different between the sets $U_{\min }$ and $U_{\max }$. The bolded entries reflect the 
decision variables that yield the five smallest $p$-values when comparing means between the two groups. The minimum cardinality $(m)$, the number of single element rows $\left(x_{3}\right)$, the proportion of nonzero entries in $A A^{T}\left(x_{8}\right)$, the standard deviation of column sum $\left(x_{21}\right)$, and the max column sum $\left(x_{25}\right)$ are among the most statistically different variables between the two sets $U_{\min }$ and $U_{\max }$.

Minimum cardinality is a strong factor for determining the number of alternative solutions. Figure 2 compares the number of alternative optimal solutions for each cardinality using a series of boxplots. The red crosses denote outliers in the respective set of matrices for each cardinality. The number of solutions has a strong right skew for each cardinality. The majority of the matrices with minimum cardinality of three have less than five solutions with a maximum of 24 .

In contrast, matrices with minimum cardinality of five or six can contain solutions in the hundreds. In all cases, however, at least one unique solution is attained for all minimum cardinalities. It provides insight into the fact that minimum cardinality will be an important predictor variable in determining the number of alternative solutions.

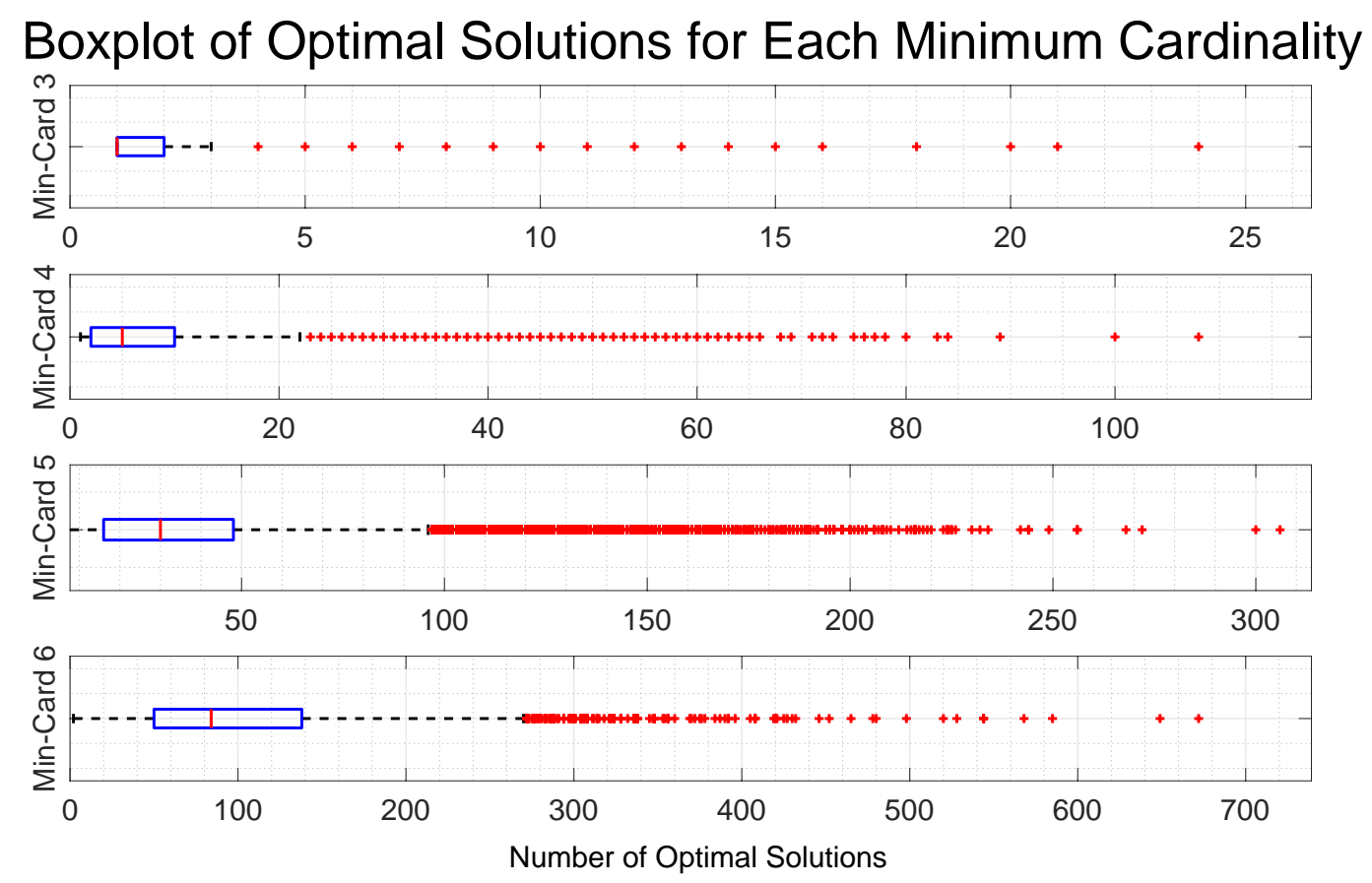

Figure 2. A series of boxplots for each set of optimal solutions according to minimum cardinality

\subsection{Regression Tree Analysis}

Categorical Regression Trees were constructed using the built-in fitctree function in MATLAB from the Statistical and Machine Learning Toolbox. This function will create a regression decision tree trained from each of the matrices corresponding to the 600,000 MCSCPs. Each input to construct a tree will consist of a matrix with attributes from Table 2. These variables will act as standard classification and regression trees (CART) predictor variables. The algorithm selects the split predictor that maximizes the Gini diversity index (GDI) that gain over all other predictors' possible splits to choose the best split predictor variable at each node. Once the tree is formed, 
the output consists of the number of solutions defined as a small, medium or large version. The algorithm creates an estimated optimal sequence of subtrees as it is growing the classification tree. In this sense, the tree can be "pruned" to contain a smaller number of nodal splits according to this optimal sequence. The subtrees are based on maximizing the GDI index at each stage (MATLAB, 2020). The tree is pruned to have the minimum number of branches such that all types of solution categories are present. We considered two regression trees with a different number of output categories.

We create two models based on the number of outputs: a 3-output model with small $(S)$, medium $(M)$, and large $(L)$ number of alternative solutions and a 5-output model with $X S, S, M, L$, $X L$, where $X S$ denotes extra small and $X L$ represents the extra-large number of alternative solutions. The discrete cutoff values for each category are determined by the criteria defined in Table 3. The interval definitions are presented in interval notation. For example, the 3-output regression tree outputs a $S$ if the tree predicts the number of solutions to be less than 6 , an $M$ if the number of solutions is predicted to be between 6 and 36 , and $L$ if the number of solutions is 36 or more. Similarly, in the 5-output regression tree, an $X S$ is for a unique solution, $S$ is for solutions between 2 and $6, M$ is for solutions between 6 and $16, L$ is between 16 and 36 , and $X L$ is beyond 36 solutions.

Table 3. Interval definitions for the categories associated to $S, M$, and $L$ for the 3-output regression trees and the categories associated to $X S, S, M, L$, and $X L$, for the 5-output regression trees

\begin{tabular}{|c|c|c|c|c|c|c|c|}
\hline \multicolumn{3}{|c|}{ 3-Output Tree } & \multicolumn{5}{c|}{ 5-Output Tree } \\
\hline$S$ & $M$ & $L$ & $X S$ & $S$ & $M$ & $L$ & $X L$ \\
\hline$[1,6)$ & {$[6,36)$} & {$[36,672]$} & {$[1,2)$} & {$[2,6)$} & {$[6,16)$} & {$[16,36)$} & {$[36,672]$} \\
\hline
\end{tabular}

Two unique regression trees are trained using the set of 600,000 matrices with all 26 predictor variables from Table 2. The unpruned regression trees each have the maximum number of branches allowed by MATLAB - 9,999 branches. The unpruned trees are not shown. These two regression trees are tested on the original data set to determine the success rate. The regression trees were pruned to have the smallest number of branch points while still maintaining every possible categorical output. The pruning technique is based on the estimated optimal sequence of subtrees. The pruned trees each have only three branches and are shown in Figure 3.

It is interesting to note the success rates for the pruned trees as well as the number of branches on the pruned trees. The unpruned trees do perform undeniably much better than the pruned trees. This is likely because the unpruned trees have a total of 26 predictor variables to help make an informed decision on whether to split into a different category or not. However, we see that there are only three branches for each of the 3-output and 5-output trees with the most important predictor variables being the number of single element rows $\left(x_{3}\right)$ and the proportion of nonzero entries in the matrix $A A^{T}\left(x_{8}\right)$. The proportion of nonzero elements in $A A^{T}\left(x_{8}\right)$ is a measure of how compatible any two columns are at covering a particular row. Indeed, if $x_{8}$ is closer to 1 , then there likely exists two columns that potentially cover identical rows, thus allowing for more alternative solutions. We note that the minimum cardinality determines the initial split. The matrix's minimum cardinality clearly distinguishes two cohorts: a smaller set of alternative 
solutions is characteristic of matrices with minimum cardinality of 4 or lower, and a larger set is typical of matrices with minimum cardinality of 5 or more. Also, $x_{3}$ decides between $M$ and $L$ for the 3-output tree and $L$ and $X L$ for the 5-output tree. Essentially, if the matrix has a single element row, it will have many solutions. Further, we see that the two trees are identical, except for the final decision categories. The success rate of both the unpruned and pruned regression trees is shown in Table 4. We see that the success rates drop dramatically for the pruned trees, but each tree is still very accurate to within one category (i.e., "off-by-one" success), with the 3-output tree being $99.93 \%$ and the 5-output tree being $94.63 \%$ accurate. Overall, it is interesting to see that only two decision variables are used with three branch points to determine the number of alternative solutions with some degree of confidence.
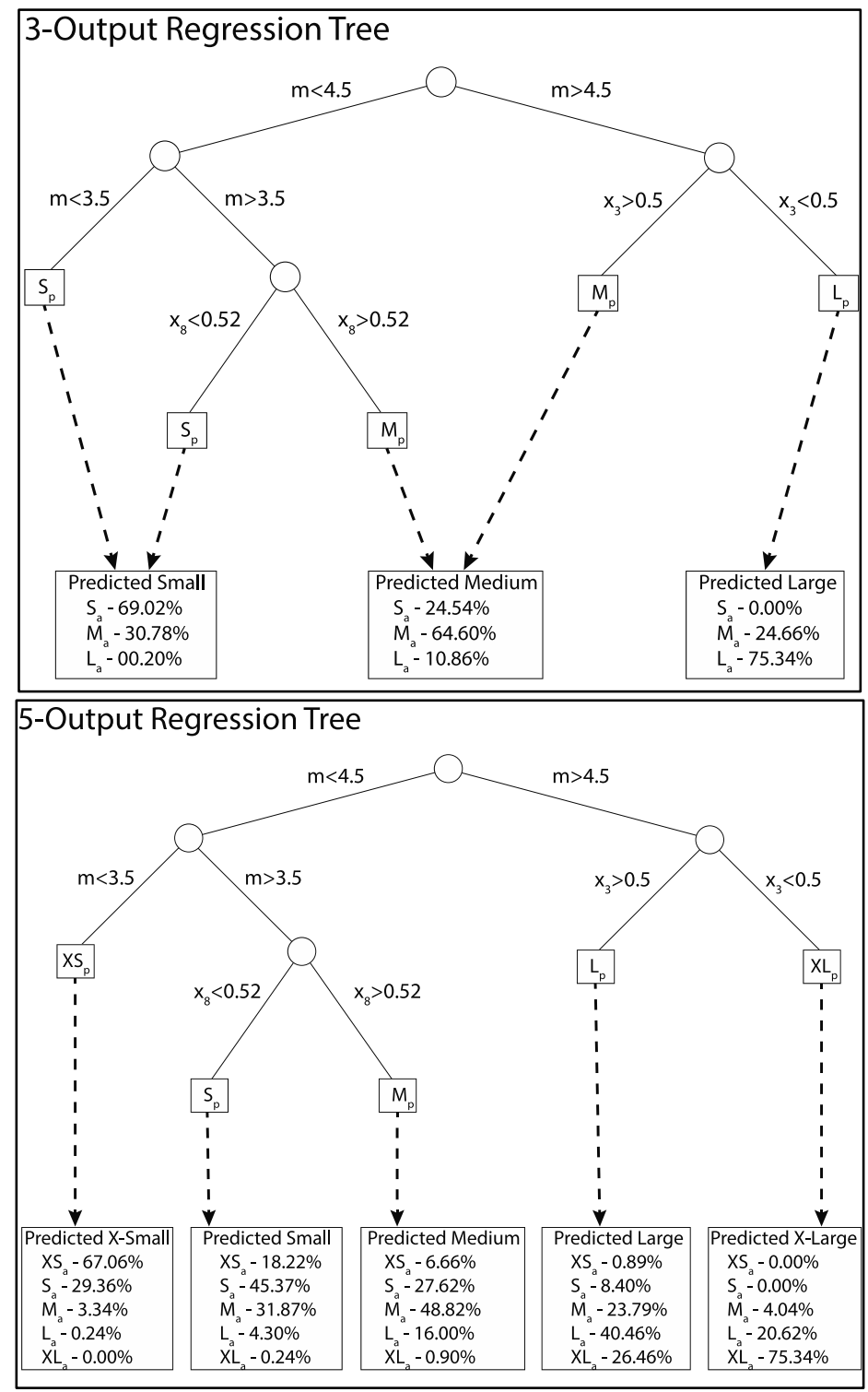

Figure 3. The 3-output and 5-output pruned regression trees 
Table 4. The success rate of the two regression trees

\begin{tabular}{|l|c|c|c|c|c|}
\hline \multirow{2}{*}{} & \multicolumn{2}{|c|}{ Not Pruned } & \multicolumn{3}{c|}{ Pruned } \\
\cline { 2 - 6 } & Success & "Off-by-One" & Success & "Off-by-One" & Branches \\
\hline 3-Output Tree & $91.50 \%$ & $99.94 \%$ & $68.59 \%$ & $99.93 \%$ & 3 \\
\hline 5-Output Tree & $84.09 \%$ & $96.97 \%$ & $50.29 \%$ & $94.63 \%$ & 3 \\
\hline
\end{tabular}

\subsection{Validation}

The two regression tree models were tested on a set of randomly generated matrices. Specifically, 5,000 matrices unique from the original set of 600,000 matrices used to train the regression tree were used to validate the two regression trees. Tables 5 and 6 show a detailed analysis of each tree's success rate for each category. For example, for the 3-output tree, the numbers in the cell labeled as actually small (denoted by the event $S_{a}$ ) and predicted small (denoted by the event $S_{p}$ ) indicated that 1,751 of the 5,000 test matrices $(35.02 \%)$ were predicted to be small by the tree and they were small. However, 781 matrices were predicted to be small, but they were medium. Considering the matrix of values, it is clear that the tree is $99.90 \%$ accurate to within one category because the only time the tree's prediction was more than one category removed was for the 5 times that the tree predicted small, but they were actually large (top right cell of the table).

Table 5. The frequencies and relative frequencies of MCSCPs that were actually $S / M / L$ and predicted to be $S / M / L$ by the pruned 3-output tree on the validation set of 5,000 MCSCPs.

\begin{tabular}{|c|c|c|c|c|c|}
\hline \multicolumn{6}{|c|}{ 3-Output Tree } \\
\hline & & \multicolumn{3}{|c|}{ Actual } & \multirow{2}{*}{ Total } \\
\hline & & $S_{a}$ & $M_{a}$ & $L_{a}$ & \\
\hline \multirow{4}{*}{$\begin{array}{l}\frac{0}{d} \\
\frac{d}{0} \\
\frac{0}{0} \\
\frac{0}{0}\end{array}$} & $S_{p}$ & $1751(35.02 \%)$ & $781(15.62 \%)$ & $5(0.10 \%)$ & $2537(50.74 \%)$ \\
\hline & $M_{p}$ & 495 (9.90\%) & $1303(26.06 \%)$ & $219(4.38 \%)$ & 2017 (40.34\%) \\
\hline & $L_{p}$ & $0(0 \%)$ & $110(2.20 \%)$ & $336(6.72 \%)$ & 446 (8.92\%) \\
\hline & Total & 2246 (44.92\%) & 2194 (43.88\%) & 560 (11.20\%) & $5000(100 \%)$ \\
\hline
\end{tabular}


Table 6. The frequencies and relative frequencies of MCSCPs that were actually $X S / S / M / L / X L$ and predicted to be $X S / S / M / L / X L$ by the pruned 5-output tree on the validation set of 5,000 MCSCPs.

\begin{tabular}{|c|c|c|c|c|c|c|c|}
\hline \multicolumn{8}{|c|}{ 5-Output Tree } \\
\hline & & \multicolumn{5}{|c|}{ Actual } & \multirow[b]{2}{*}{ Total } \\
\hline & & $X S_{a}$ & $S_{a}$ & $M_{a}$ & $L_{a}$ & $X L_{a}$ & \\
\hline \multirow{6}{*}{$\begin{array}{l}\frac{.}{\Phi} \\
\frac{0}{0} \\
\frac{0}{0} \\
0\end{array}$} & $X S_{p}$ & $\begin{array}{c}281 \\
(5.62 \%)\end{array}$ & $\begin{array}{c}123 \\
(2.46 \%)\end{array}$ & $14(0.28 \%)$ & $1(0.02 \%)$ & $0(0 \%)$ & 419 (8.38\%) \\
\hline & $S_{p}$ & $\begin{array}{c}386 \\
(7.72 \%)\end{array}$ & $\begin{array}{c}961 \\
(19.22 \%)\end{array}$ & $\begin{array}{c}675 \\
(13.50 \%)\end{array}$ & $\begin{array}{c}91 \\
(1.82 \%)\end{array}$ & $5(0.10 \%)$ & 2118 (42.36\%) \\
\hline & $M_{p}$ & $\begin{array}{c}82 \\
(1.64 \%)\end{array}$ & $\begin{array}{c}340 \\
(6.80 \%)\end{array}$ & $\begin{array}{c}601 \\
(12.02 \%)\end{array}$ & $\begin{array}{c}197 \\
(3.94 \%)\end{array}$ & $\begin{array}{c}11 \\
(0.22 \%)\end{array}$ & $1231(24.62 \%)$ \\
\hline & $L_{p}$ & $7(0.14 \%)$ & $\begin{array}{c}66 \\
(1.32 \%)\end{array}$ & $\begin{array}{c}187 \\
(3.74 \%)\end{array}$ & $\begin{array}{c}318 \\
(6.36 \%)\end{array}$ & $\begin{array}{c}208 \\
(4.16 \%)\end{array}$ & $786(15.72 \%)$ \\
\hline & $X L_{p}$ & $0(0 \%)$ & $0(0 \%)$ & $18(0.36 \%)$ & $\begin{array}{c}92 \\
(1.84 \%)\end{array}$ & $\begin{array}{c}336 \\
(6.72 \%)\end{array}$ & 446 (8.92\%) \\
\hline & Total & $\begin{array}{c}756 \\
(15.12 \%)\end{array}$ & $\begin{array}{c}1490 \\
(29.80 \%)\end{array}$ & $\begin{array}{c}1495 \\
(29.90 \%)\end{array}$ & $\begin{array}{c}699 \\
(13.98 \%)\end{array}$ & $\begin{array}{c}560 \\
(11.20 \%)\end{array}$ & $5000(100 \%)$ \\
\hline
\end{tabular}

\section{5-Output Tree}

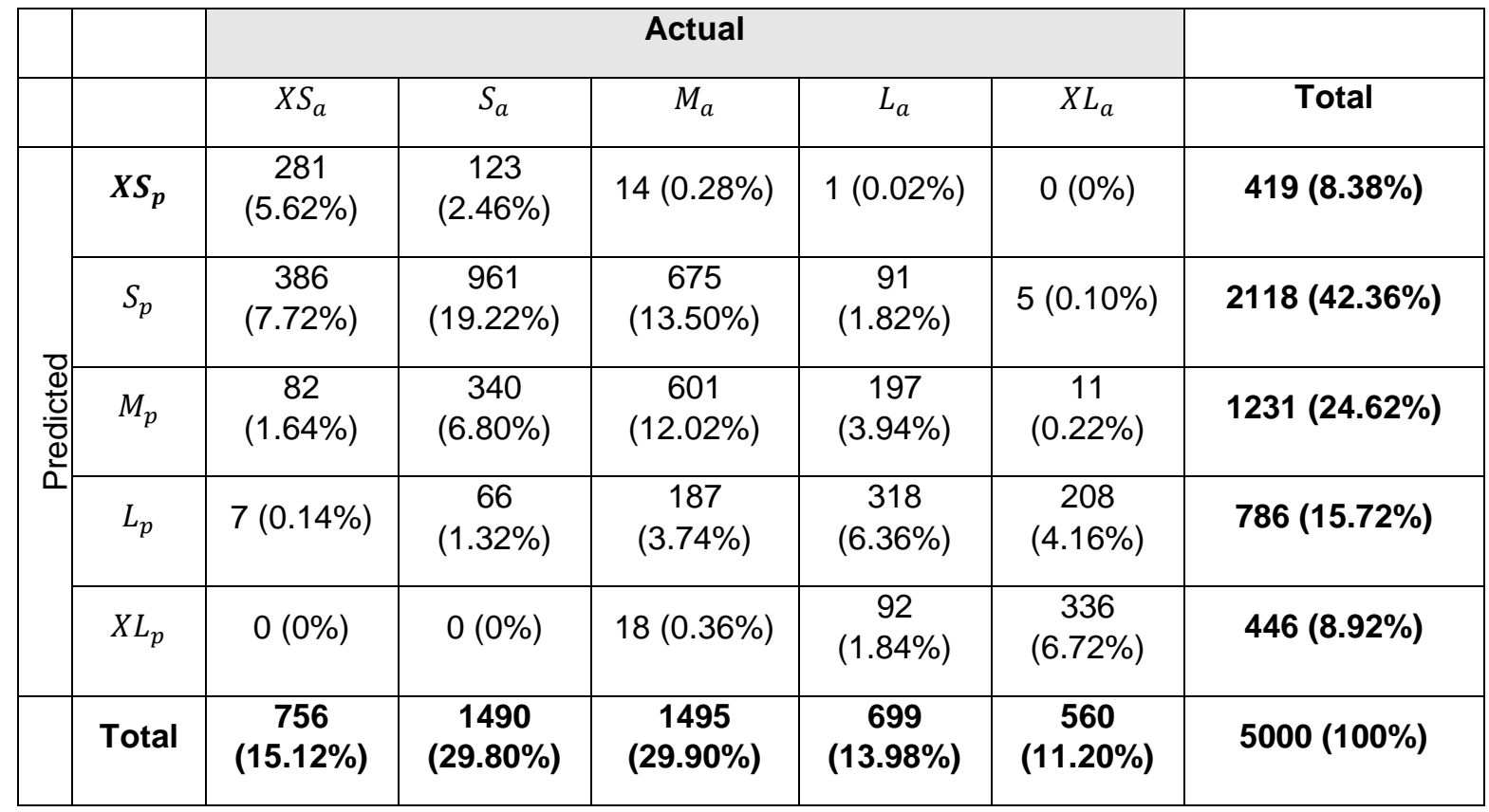


Using this information, the 3-output and 5-output regression trees are presented in Figure 3 with the final decision, including weighted percentages of the tree's misreading. Conditionally, for the 5 -output model, if we know the tree has predicted extra-large, there is a $75.34 \%$ chance (based on this simulation of 5,000 random matrices) that the matrix actually has an extra-large number of alternative solutions, i.e.,

$$
P\left(X L_{a} \mid X L_{p}\right)=\frac{336}{0+0+18+92+336}=75.34 \% .
$$

We may also define the "off-by-one" conditional probability of success. For instance, let $\widetilde{L_{a}}$ denote the event that the MCSCP is within one category of large, i.e., it either has medium, large, or extra-large alternative optima. The, we may calculate the following probability to indicate that if the 5-output model predicts a large number of solutions, then we can be $90.71 \%$ confident that the actual number of solutions is within one category of being large, based on this set of 5,000 test matrices.

$$
P\left(\widetilde{L_{a}} \mid L_{p}\right)=\frac{187+318+208}{7+66+187+318+208}=90.71 \%
$$

\section{Results and Discussion}

Our preceding analysis yields several important results that shed light on the idea of predicting the number of alternative solutions to the MCSCP. The statistical work conducted above on a set of 600,000 randomized MCSCPs gives insight into the distribution of alternative optima. Our representative sample found that the majority, $90.04 \%$, of MCSCPs of size $10 \times 20$ with $20 \%$ density of ones has a minimum cardinality of 4 or 5 . For the whole representative sample, we find that the distribution of the number of alternative solutions is severely skewed, with 13.76 being the average number of alternative optima with a maximum number of 672. An MCSCP is considered an outlier relative to the data if it has greater than 35 alternative optima. Approximately $10 \%$ of the data are outliers, and approximately $15 \%$ of MCSCPs considered have a unique solution. Given the data, every outlier has a minimum cardinality of at least 4 . It indicates that minimum cardinality is a reliable determinant of a larger number of alternative optima, resulting from our regression tree analysis. Our set of 600,000 matrices, although only a small proportion of the possible problems of size $10 \times 20$, illustrates the nature of how alternative optima arise in MCSCPs. Constructing the population of all MCSCPs of this size is unfathomable, and thus our statistical approach using a randomized sample of a decent size provides a first look into the nature of these problems.

The two pruned regression trees, presented in Figure 3, help to predict, at least qualitatively, the number of alternative optima for any given MCSCP. The unpruned trees perform extraordinarily well on the original training set, but they have an unreasonable number of branches. Therefore, they would likely not be used in practice. The pruned trees still perform quite well with only three branches and provide insight into the most important characteristics of the MCSCP. Indeed, the decision nodes determined by the regression tree algorithm indicate that minimum cardinality $(m)$, the number of single element rows $\left(x_{3}\right)$, and the proportion of non-zeros in the matrix $A A^{T}\left(x_{8}\right)$ have the most impact. Given any MCSCP, these three numerical values are not difficult to compute and yet, based on our analysis, should give a reasonable prediction 
about the number of alternative optima. We find that if the minimum cardinality is greater than 4 , then it is probable that the MCSCP has a medium, large, or extra-large number of optimal solutions. In the 3-output model, this means that the MCSCP has at least 6 alternatives optimal. Using the 5-output model, this means the problem has at least 16 alternative optima. In any case, we can conclude that if the minimum cardinality is more than 4 , the problem likely does not have a unique solution. In contrast, our models show that if the minimal cardinality is less than 3 , the MCSCP probably has less than 5 alternative solutions.

A more in-depth look into our regression trees show that the variables $x_{3}$ and $x_{8}$ are the deciding factors in the second phase of the algorithm. Indeed, the number of single-row elements typically determines the difference between medium and large or large and extra-large for the 3output and 5-output models, respectively. This is a reasonable result because if an MCSCP has a larger minimal cardinality, the number of solutions will only increase if there are less "isolated" points. Interestingly, we find that if a matrix has one or more single element row, then this will significantly change the number of alternative solutions. According to both models, if the matrix has zero single element rows, it is likely an outlier (in the sense of alternative optima). Likewise, if the proportion of nonzero elements of $A A^{T}$ is less than 0.52 , then the problem likely has a small number of solutions rather than a medium number. Of the 26 possible decision variables considered, we find that only these two are the most important for determining more subtle differences in the number of alternative optima.

We validated the two regression trees on a unique set of 5,000 MCSCPs. The preliminary results of the validation are shown in Figures 5 and 6 . Using the validation, we can provide more insight into our models' success and assign probabilities to possible outcomes. Although the pruned trees do not effectively predict exact matches on the original training set $(68.59 \%$ for 3 output and $50.29 \%$ for 5 -output), we find that the pruned models do perform well for predicting the number of solutions to within one category (99.93\% for 3-Output and $94.63 \%$ for 5 -output). On the validation set, we see that both models perform best when predicting exact matches for large optima numbers. That is, $P\left(L_{a} \mid L_{p}\right)=75.34 \%$ for the 3-output model and $P\left(X L_{a} \mid X L_{p}\right)=$ $75.34 \%$ for the 5 -output model. The 3 -output model manages to maintain more than $60 \%$ accuracy for exact small and medium predictions. If we relax the definition of success and consider correct predictions within one category (i.e., "off-by-one" success), we see that both models reach at least $90 \%$. Indeed, using the output from Table 6 , we may compute the conditional probabilities in Table 7, which show the performance of the 5-output regression tree in determining the number of optimal solutions to within one category. In this case, the average success rate is $94.20 \%$, which indicates that the 5-output is successful in predicting, at least qualitatively, the number of alternative optima of a MCSCP.

Table 7. The performance of the 5-output tree in predicting the correct number of alternative solutions

\begin{tabular}{|c|c|}
\hline Conditional Probability & "Off-by-One" Success \\
\hline$P\left(\widetilde{X S_{a}} \mid X S_{p}\right)$ & $96.42 \%$ \\
\hline$P\left(\widetilde{S_{a}} \mid S_{p}\right)$ & $95.48 \%$ \\
\hline$P\left(\widetilde{M_{a}} \mid M_{p}\right)$ & $92.45 \%$ \\
\hline$P\left(\widetilde{L_{a}} \mid L_{p}\right)$ & $90.71 \%$ \\
\hline$P\left(\widetilde{X L_{a}} \mid X L_{p}\right)$ & $95.96 \%$ \\
\hline
\end{tabular}


Our present analysis uses the minimum cardinality $(m)$ as an input variable to train the regression tree. It comes as no surprise that this variable is the most important in determining each tree's initial decision node. To isolate this variable, we also created regression trees within each minimum cardinality (not shown). We found that not only were $x_{3}$ and $x_{8}$ important variables within each cardinality, but also the standard deviation of the row sum $\left(x_{15}\right)$. We see similar accuracies for regression trees within each minimum cardinality.

\section{Conclusion}

The present study's goal was to qualitatively predict the number of alternative optima for a classic NP-hard combinatorial optimization problem such as the MCSCP. To the authors' knowledge, this article is the first attempt to answer this question. Aside from being an interesting theoretical question, the answer to this question has potential practical implications. Our methods to randomly generate matrices corresponding to 600,000 MCSCPs were analyzed using the machine learning function of MATLAB®. Twenty-six matrix characteristics were identified as potentially relevant for this analysis and used as input to generate categorical regression trees. There were two regression trees generated: a 3-output tree and a 5-output tree. The 3-output tree predicted either a small, medium, or large number of solutions for an MCSCP, and the 5-output tree predicted either an extra small, small, medium, large, or extra-large number of solutions for an MCSCP. The prediction quality of these trees was determined using a separate set of 5,000 MCSCPs. The trees were most accurate in predicting a large number (3-output) of optimal solutions and an extra-large number (5-output) of optimal solutions. We find that both models are particularly accurate in predicting whether an MCSCP has a large number of solutions based on only three, easily calculable characteristics of the constraint matrix. Indeed, the 5-output model can essentially predict the nature of the number of alternative optima within one category with a success rate of $94.20 \%$, on average.

The significance of this study is observed in the potential applications it has to real-world problems. One specific application is that of ingot mold selection (Vasko et al., 1987). The goal of such a study is to determine the minimum number of ingot mold sizes. Once the minimum number of ingot mold sizes is found, the secondary objective is to minimize yield loss. Using our methodology, if the application is predicted to have a small number of alternative optima, then more effort may be required to find a near-optimal solution for the second objective function. Alternatively, if our methodology predicts that many alternative solutions exist, then it may require less computational effort to find a near-optimal solution for the secondary objective.

This work was a first attempt to qualitatively predict the number of optimal solutions to a MCSCP, so our results are limited to a specific MCSCP, one that corresponds to a constraint matrix of size $10 \times 20$ with $20 \%$ density of ones. Therefore, the results of this analysis may be regarded as a pilot study for problems of this nature. Given the success obtained so far, future work will involve using a larger variety of MCSCPs as input to generate models that qualitatively predict the number of optimal MCSCP solutions. We seek to expand the results presented here to problems with different densities of ones and/or larger sizes in general. We may be able to speculate that keeping the size the same while increasing the density will yield a larger proportion of MCSCPs with lesser minimum cardinalities, thereby decreasing the number of alternative solutions across the board. We wish to determine specific trends in MCSCPs with varying densities as well as with varying sizes. However, as the constraint matrix size increases, the curse 
of dimensionality may limit our progress since searching for all optimal solutions of a specific cardinality becomes a daunting task. Finally, we are interested in applying the methodology discussed in this article to analyze the number of alternative optima for other combinatorial optimization problems.

\section{References}

C. Saleh, R.A.C. Leuuveano, M.N.A. Rahman, B. M. Deros, \& N.R., Dzakiyullah. (2015). Predicting of $\mathrm{CO}_{2}$ emissions using an artificial neural network: the case of the sugar industry. Adv. Sci. Lett., 21, 3079-3083.

D. K. Williams Jr., A. L. Kovach, D. C. Muddiman, \& K. W. Hanck. (2009). Utilizing artificial neural networks in MATLAB to achieve parts-per-billion mass measurement accuracy with a fourier transform ion cyclotron resonance mass spectrometer. American Society for Mass Spectrometry, 20, 1301-1310.

E.L. Lawler. (1972). Procedure for computing the K best solutions to discrete optimization problems and its application to the shortest path problem. Management Science, 18(7), 401405.

F. J. Vasko, F. E. Wolf, \& K. L. Stott. (1987). Optimal selection of ingot sizes via set covering. Opns Res, 35, 346-353.

F. J. Vasko, F. E. Wolf, \& K. L. Stott. (1989). A set covering approach to metallurgical grade Assignment. Eur J Opl Res, 38, 27-34.

F. J. Vasko, D. D. Newhart, \& A. D. Strauss. (2005). Coal blending models for optimum cokemaking and blast furnace operation. Journal of the Operational Research Society, 56 (3), 235-243.

F. S. Hillier \& J. Lieberman. (2010). Introduction to Operations Research. $9^{\text {th }}$ Edition, New York: McGraw-Hill.

H.W. Hamacher \& M. Queyranne, M. (1985). K-best solutions to combinatorial optimization problems. Annals of Operations research, 4, 123-145.

H. Taha. (2017). Operations Research: An Introduction, $10^{\text {th }}$ Edition, Pearson, Boston, MA.

MATLAB. (2020). Statistics and Machine Learning Toolbox User's Guide. R2020a, 1 Apple Hill Dr, Natick, MA 01760-2098.

R. E., Bixby. (2012). A brief history of linear and mixed-integer programming computation. Documenta Mathematica Extra volume ISMP, 107-121.

R. M. Karp. (1972). Reducibility among combinatorial problems," in Complexity of Computer Computations, Miller R E and Thatcher J W (eds), 85-103. Plenum, New York.

T. Huang, Y. Gong, \& J. Zhang. (2018). Seeking multiple solutions of combinatorial optimization problems: a proof of principle study", in 2018 IEEE Symposium Series on Computational Intelligence (SSCl), 1212-1218. Bangalore, India. 
This page is intentionally left blank. 